Original Article

\title{
EFFECTS OF TAMOXIFEN OR LETROZOLE ON LIPID PROFILE, VITAMIN D AND ESTRADIOL SERUM LEVELS IN OBESE POSTMENOPAUSAL WOMAN WITH BREAST CANCER
}

\author{
HAEDER ABDULHAFITH AL-BIATI ${ }^{1}$, AHMED SALIH SAHIB ${ }^{2 *}$, ANWAR NOORI MAHMOOD ${ }^{3}$ \\ ${ }^{1}$ Department of Clinical Pharmacy, College of Pharmacy, University of Bagdad, Iraq, ${ }^{2}$ Department of Pharmacology, College of Pharmacy, \\ University of Kerbala, Iraq, ${ }^{3}$ Department of Surgery, Baquba Teaching Hospital, Diyala, Iraq \\ Email: ahmedsalih73@yahoo.com \\ Received: 27 Aug 2016 Revised and Accepted: 21 Dec 2016
}

\begin{abstract}
Objective: Obesity is associated with both increased breast cancer risk and poorer prognosis after disease onset. Women who are obese continue to have higher levels of estrogen than women of normal weight even after treatment with hormone-suppressing drugs, raising the possibility that they might benefit from modification or changes to their treatment. The aim of this work was to study the effect of letrozole compared to tamoxifen on serum estradiol and, vitamine D and metabolic profile in Iraqi obese postmenopausal women with breast cancer.
\end{abstract}

Methods: A hospital-based case-control study was carried out at Baquba teaching hospital, Diyala, Iraq. The analyzed variables were: age, lipid profile including total cholesterol; triglycerides, HDL-C, blood sugar, estradiol, and serum vit D. Descriptive statistics and testing of hypothesis were used for the analysis using mean $\pm S D$ test $\mathrm{P} \leq 0.05$.

Results: Lipid profile, serum estradiol showed significant variability among the studied group in this study and serum vit D show significant differences between groups in postmenopausal obese with breast cancer taking tamoxifen.

Conclusion: Treatment of obese women with breast cancer with tamoxifen or letrozole had neglected effects on metabolic parameters including lipid profile and blood sugar, both agents decrease serum estradiol level in treated patients, and most importantly, the significant positive effect of tamoxifen on serum vitamin D level compared to negative effect of aromatase inhibitor drug letrozole.

Keywords: Breast cancer, Postmenopausal, Vitamin D, Estradiol

(C) 2017 The Authors. Published by Innovare Academic Sciences Pvt Ltd. This is an open access article under the CC BY license (http://creativecommons.org/licenses/by/4. 0/] DOI: http://dx.doi.org/10.22159/ijpps.2017v9i2.14918

\section{INTRODUCTION}

Breast cancer is a worldwide major public health problem in women population, affecting both the developing and developed countries and comprising $18 \%$ of all female cancers; more than 1.2 million cases are diagnosed every year, affecting $10-12 \%$ of the female population and accounting 500,000 deaths per year worldwide [1]. It is well established that women having low-income and worse socioeconomic conditions are at increased risk to develop breast cancer and have lower rates of survival in already existed breast cancer [2]. Reporting of variation in incidence of breast cancer in different population of different parts of Asian continent may be due to multiple factors, including geographic variation, racial/ethnic background, genetic variation, lifestyle, environmental factors, socioeconomic status, the presence of known risk factors, and utilization of screening mammography, stage of disease at diagnosis, and the availability of appropriate care [3]. Breast cancer is the most common cancer among Iraqi women, especially in the last ten years; it has been reported that breast cancer is the commonest type of female malignancy, accounting for approximately $30 \%$ of the registered female cancers according to the latest Iraqi Cancer Registry [4]. Obesity is associated with both increased breast cancer risk and poorer prognosis after disease onset [5].

Another risk factor for breast cancer is a deficiency of 25-OH vitamin D. Experimental studies have shown that 1, $25(\mathrm{OH}) 2 \mathrm{D}$ can inhibit cellular proliferation, induces differentiation and apoptosis, and inhibit angiogenesis in normal and malignant breast cells [6, 7]. In rodent models, high intake of vitamin $\mathrm{D}$ has been shown to suppress high-fat-diet-induced epithelial hyperproliferation and tumor genesis of the mammary gland [8]. In addition, a nongenomic pathway of 1, $25(\mathrm{OH}) 2 \mathrm{D}$ has been shown, in which 1, $25(\mathrm{OH}) 2 \mathrm{D}$ interacts largely with membrane VDR to exert its biological effects by altering intracellular calcium channels [9]. However, little is known about the effect of obesity on treatment efficacy. Women who are obese continue to have higher levels of oestrogen than women of normal weight even after treatment with hormone-suppressing drugs, raising the possibility that they might benefit from modification or changes to their treatment $[10,11]$. The aim of this work was to study the effect of letrozole compared with tamoxifen on serum level of estrodiol, vitamin D level and other biochemical parameters in Iraqi obese postmenopausal patients with breast cancer.

\section{MATERIALS AND METHODS}

\section{Methods}

This study was carried out at Baquba teaching hospital, Diyala, Iraq; and approved by local institutional scientific and ethical committee; an informed consent was taken from all participants; 30 postmenopausal women newly diagnosed with breast cancer, who were visited the consultation clinic participated in the study. Patients are suffering from any other cancer as well as diabetes mellitus and dyslipidemia was excluded from the study. A mammography and histopathological section were done which showed changes of well differentiated intracystic papillary carcinoma with areas showing an infiltration carcinoma invading stoma with differing grade and no vascular invasion or estrogen and positive progesterone tests. The patients have divided into two parts 15 obese postmenopausal women on Tamoxifen $20 \mathrm{mg}$ once daily for three months and 15 obese women on Letrozole $2.5 \mathrm{mg}$ once daily for three months in comparison with 10 control postmenopausal women without breast cancer. An informed consent was taken from all participants. From each patient and control, blood samples were collected before and after three months. The collected and analysed variables were age, body mass index, the metabolic profile including total cholesterol, triglycerides, HDL-C, blood sugar, serum estradiol and serum 25-OHvitamine D were compared for two groups. All parameters were analysed in the laboratory of Baquba teaching hospital and AL-shams laboratory using Human reagent kits on semi auto analyser (Humalyser 3500), Minividus elisa for measuring estradiol. Vit. D was measured by elisa kit. 


\section{Statistical analysis}

The data were subjected to statistical analysis using the student t-test for comparison of means between studied groups, utilising SPSS 18 version. All the data were expressed as mean \pm standard deviation of the mean. P-Value $\leq 0.05$ were considered significant.

\section{RESULTS}

Table 1 showed the comparison of age and body mass index between groups included in this study. There is no significant difference in the age of the studied groups, meanwhile; results showed significant difference concerning BMI in the studied groups after three months of treatment when compared to baseline values.

Table 1: Demographic data of participants

\begin{tabular}{llllll}
\hline Variables & Group 1 n=10 & Group 2 n=15 & Group 3 n=15 & 3 mo \\
\hline & & Baseline & $\mathbf{3 ~ m o}$ & Baseline & - \\
\hline Age (years) & $54.7 \pm 3.47$ & $54.3 \pm 3.47$ & - & $53.8 \pm 2.66$ & $30.038 \pm 1.74$ \\
BMI $\left(\mathrm{Kg} / \mathrm{m}^{2}\right)$ & $28.56 \pm 1.45$ & $28.1 \pm 1.8$ & $27.12 \pm 1.21^{*}$ & $29.56 \pm 1.74^{*}$ & \\
\hline
\end{tabular}

Results represent mean \pm SD; Group 1: Control, Group 2: Obese postmenopausal women with breast cancer treated with tamoxifen 20 mg daily. Group 3: Obese postmenopausal women with breast cancer treated with letrozole $2.5 \mathrm{mg}$ daily. $\mathrm{P} \leq 0.05$ considered significant*.

Table 2 showed the changes in fasting blood sugars and lipid profile values among studied groups, there was no significant change in the serum level of total cholesterol between the studied groups when comparing with control group and with baseline values; concerning serum level of triglycerides, the value of studied groups at baseline were significantly higher than the value of control group. The baseline serum level of high-density lipoprotein of group 3 was significantly lower than that of both groups 1 and 2 . Finally the baseline value of fasting blood sugar of tamoxifen-treated group was significantly higher than that of both control and letrozole treated groups.

Table 2: Lipid profile and fasting blood sugar levels of studied subjects

\begin{tabular}{lllll}
\hline Variables & Group 1 n=10 & Group 2 n=15 & & Group 3 n=15 \\
\cline { 2 - 4 } & & Baseline & 3 mo & Baseline \\
\hline S. TCh mg/dl & $207.54 \pm 5.76$ & $223.6 \pm 42.7$ & $212.45 \pm 26.45$ & $198.7 \pm 15.36$ \\
S. TG mg/dl & $170.2 \pm 20.96$ & $304.5 \pm 15.2^{*}$ & $341.21 \pm 12.65$ & $215.65 \pm 7.3^{*}$ \\
S. HDL mg/dl & $50.2 \pm 8.61$ & $48.32 \pm 4.6$ & $51.4 \pm 5.76$ & $205.45 \pm 7.15$ \\
FBS mg/dl & $113.1 \pm 8.99$ & $192.1 \pm 2.44^{*}$ & $187.5 \pm 8.7$ & $34.4 \pm 1.96^{*}$ \\
\hline
\end{tabular}

Results represent mean \pm SD; Group 1: Control, Group 2: Obese postmenopausal women with breast cancer treated with tamoxifen 20 mg daily. Group 3: Obese postmenopausal women with breast cancer treated with letrozole $2.5 \mathrm{mg}$ daily. $\mathrm{P} \leq 0.05$ considered significant*.

Table 3 showed that serum estradiol level was significantly ( $\mathrm{p} \leq 0.05)$ decreased in patients with breast cancer taking tamoxifen or letrozole for three months compared to baseline values, while serum vit. D level significantly $(\mathrm{p} \leq 0.05)$ increased after three months of tamoxifen-treated group compared to baseline value, on the contrary, letrozole treated group showed a significantly reduction in serum vit. D level after three months of treatment compared to baseline value.

Table 3: Serum estradiol and vit. D (25-OH) levels in studied subjects

\begin{tabular}{lllll}
\hline Variables & Group 1 n=10 & Group 2 n=15 & & \multicolumn{2}{c}{ Group 3 n=15 } \\
\cline { 3 - 5 } & & Baseline & 3 mo & Baseline \\
\hline S. Estradiol pg/ml & $15.67 \pm 2.1$ & $23.61 \pm 2.54$ & $19.45 \pm 1.34^{*}$ & $29.45 \pm 2.98$ \\
S. Vitamin-D ng/ml & $30.23 \pm 3.1$ & $27.61 \pm 1.64$ & $28.35 \pm 2.51^{*}$ & $28.54 . \pm 3.21$ \\
\hline
\end{tabular}

Results represent mean \pm SD; Group 1: Control, Group 2: Obese postmenopausal women with breast cancer treated with tamoxifen 20 mg daily. Group 3: Obese postmenopausal women with breast cancer treated with letrozole $2.5 \mathrm{mg}$ daily. P $\leq 0.05$ considered significant*.

\section{DISCUSSION}

The rate at which breast cancer is spreading like an epidemic is alarming and there are numerous cases reported in recent years. This observation calls for urgent control and management of this type of cancer. A decline in the cell-mediated immunity predisposes to oncogenesis, and a close association has been found between immune responses and macro or micronutrient status [12]. Table 1 showed that there was no significant difference between age groups. At the same time, BMI was significantly decreased in group 2 patients who were taking tamoxifen tablet $20 \mathrm{mg}$ for three months also in group 3 patients who were taking letrozole. This is positive worth related to BMI. The positive effect of tamoxifen on BMI may lead due to regulation on serum leptin which regulates food intake [13]. This is with an agreement with another study in ovariectomized female rats; tamoxifen led to decreased food intake, and reduced body fat and body weight [14]. Another study that with an agreement with results tamoxifen suppressed weight gain in female Wistar-Kyoto (WKY) rats, although it was not achieved only via suppression of food intake [15]. In the other study, there was disagreement with results the mechanism involved was a decrease in the FAS mRNA expression, which caused the accumulation of malonyl-CoA in the ventromedial nucleus of the hypothalamus [16] Thus, the effect of tamoxifen on energy balance, which could involve ER agonist activity in the hypothalamus, should be investigated in further studies. Table 2 showed the effect of tamoxifen and letrozole on the lipid profile of postmenopausal women after treatment for three months. Tamoxifen has a bad effect on serum triglyceride and more pronounced than letrozole $2.5 \mathrm{mg}$ once daily for three months. In other hand, good effects on serum cholesterol and serum HDL. In addition, tamoxifen causes decrease non-significantly in fasting blood sugar while letrozole causes increase significantly in fasting 
blood sugar. This is agree with other studies in which overweight women, insulin sensitivity decreased dramatically in patients taking tamoxifen compared to those on placebo [17]. The underlying mechanism by which tamoxifen increases insulin resistance remains poorly understood but could involve the development of hepatic steatosis. Interestingly, in wild-type female mice, tamoxifen reversed the protective effect of estradiol on preventing insulin-deficient diabetes induced by streptozotocin (STZ), suggesting that tamoxifen acts as an ER antagonist in pancreatic $\beta$-cells and may have adverse action in pancreatic islets [18]. Tamoxifen treatment caused severe hypertriglyceridemia in both breast cancer patients with preexisting diabetes [19] and those with no history of diabetes [20] indicating that tamoxifen usage exacerbates lipid abnormalities.

In a rat mammary tumor model induced by 7, 12-dimethylbenz[a] anthracene (DMBA), tamoxifen treatment increased the synthesis of triglyceride in the liver without affecting fatty acid b-oxidation, resulting in elevated liver triglyceride content [21]. Another report found that tamoxifen treatment increased de novo fatty acid synthesis in mouse liver, which was achieved at least partially through downregulating the activity of AMPK kinase, a master activator of fatty acid oxidation and suppressor of fatty acid synthesis [22]. The results are with an agreement with an opinion on the positive effect of tamoxifen on bone mineral density.

In comparison, both letrozole and tamoxifen had negative effects on bone mineral density and increased the risk of fractures [23]. The present study shows that letrozole $2.5 \mathrm{mg}$ induced an intense suppression of 17-estradiol at three months as compared with tamoxifen in obese postmenopausal patients with hormoneresponsive early breast cancer. This suppression of 17 -estradiol levels is expected by the mechanism of action of letrozole. Few data have been reported to date on endocrine effects of AIs in postmenopausal patients with breast cancer, and no one has compared letrozole with tamoxifen in terms of hormonal changes [24].

\section{CONCLUSION}

In conclusion, results of this study showed that treatment of obese women breast cancer with tamoxifen or letrozole had neglected effects on metabolic parameters including lipid profile and blood sugar, both agents decrease serum estradiol level in treated patients, and most importantly, the significant positive effect of tamoxifen on serum vitamin D level compared to negative effect of letrozole which results in significant reduction on serum vitamin D level.

\section{CONFLICTS OF INTERESTS}

Declared none

\section{REFERENCES}

1. Benson JR, Jatoi I, Keisch M, Esteva FJ, Makris A, Jordan VC. Early breast cancer. Lancet 2009;373:1463-79.

2. Brandt A, Bermejo JL, Sundquist J, Hemminki K. Age of onset in familial breast cancer as background data for medical surveillance. Br J Cancer 2010;102:42-7.

3. Hortobagyi GN, de la Garza Salazar J, Pritchard K, Amadori D, Haidinger R, Hudis CA, et al. The global breast cancer burden: variations in epidemiology and survival. Clin Breast Cancer 2005;6:391-401.

4. Iraqi Cancer Board. Results of the Iraqi Cancer Registry 2004. Baghdad, Iraqi Cancer Registry Center, Ministry of Health; 2007.

5. Doyle SL, Donohoe CL, Lysaght J, Reynolds JV. Visceral obesity, metabolic syndrome, insulin resistance and cancer. Proc Nutr Soc 2001;3:1-9.
6. Welsh J. Vitamin D and breast cancer: insights from animal models. Am J Clin Nutr 2004;80:1721-4S.

7. Arora H, Dixit V, Dhanwal D, Srivastava N. Vitamin D, an evidence based medicine. Innovare J Med Sci 2016;4:19-20.

8. Goyal A, Verma A, Kaur R, Kamboj A, Jain JK. The beneficial effect of vitamin D on high-fat diet-induced obesity in wistar rats. Asian J Pharm Clin Res 2016;9:337-40.

9. Mehta RG, Mehta RR. Vitamin D and cancer. J Nutr Biochem 2002;13:252-64.

10. Garrett A, Quinn MA. Hormonal therapies and gynaecological cancers. Best Pract Res Clin Obstet Gynaecol 2008;22:407-21.

11. Gould RE, Garcia AA. Update on aromatase inhibitors in breast cancer. Curr Opinion Obstetrics Gynecol 2006;18:41-6.

12. Gowal S, de Giacoms M, Le Boudec JY. A validated mathematical method of cell mediated immune response to tumor growth. Cancer Res 2007;67:8419-21.

13. Wade GN, Heller HW. Tamoxifen mimics the effects of estradiol on food intake, body weight, and body composition in rats. Am J Physiol 1993;264:R1219-23.

14. Wallen WJ, Belanger MP, Wittnich C. Sex hormones and the selective estrogen receptor modulator tamoxifen modulate weekly body weights and food intakes in adolescent and adult rats. J Nutr 2001;131:2351-7.

15. Considine RV, Sinha MK, Heiman ML, Kriauciunas A, Stephens TW, Nyce MR, et al., Serum immunoreactive-leptin concentrations in normal-weight and obese humans. N Engl J Med 1996;334:292-5.

16. Ozet A, Arpaci F, Yilmaz MI, Ayta H, Ozturk B, Komurcu S, et al. Effects of tamoxifen on the serum leptin level in patients with breast cancer. Japan J Clin Oncol 2001;31:424-7.

17. Günel N, Coşkun U, Toruner FB, Sancak B, Yilmaz E, Cengiz O, et al. Serum leptin levels are associated with tamoxifen-induced hepatic steatosis. Curr Med Res Opin 2003;19:47-3.

18. López M, Lelliott CJ, Tovar S, Kimber W, Gallego R, Virtue S, et al. Tamoxifen-induced anorexia is associated with fatty acid synthase inhibition in the ventromedial nucleus of the hypothalamus and accumulation of malonyl-Co A. Diabetes 2006;55:1327-36.

19. Vogel VG, Costantino JP, Wickerham DL, Cronin WM, Cecchini RS, Atkins JN, et al., Effects of tamoxifen vs raloxifene on the risk of developing invasive breast cancer and other disease outcomes: the NSABP study of tamoxifen and raloxifene (STAR) P-2 trial. JAMA 2006;295:2727-41.

20. Sato M, Rippy MK, Bryant HU. Raloxifene, tamoxifen, nafoxidine, or estrogen effects on reproductive and nonreproductive tissues in ovariectomized rats. FASEB J 1996;10:905-12.

21. Love R, Mazess RB, Barden HS, Epstein S, Newcomb PA, Jordan $\mathrm{VC}$, et al. Effects of tamoxifen on bone mineral density in postmenopausal women with breast cancer. N Engl J Med 1992;326:852-6.

22. Vestergaard P, Rejnmark L, Mosekilde L. Effect of tamoxifen and aromatase inhibitors on the risk of fractures in women with breast cancer. Calcif Tissue Int 2008;82:334-40.

23. Shapiro CL. Aromatase inhibitors and bone loss: risks in perspective. J Clin Oncol 2005;23:4847-9.

24. Smith IE, Dowsett M. Aromatase inhibitors in breast cancer. N Engl J Med 2003;348:2431-42.

\section{How to cite this article}

- Haeder Abdulhafith AL-Biati, Ahmed Salih Sahib, Anwar Noori Mahmood. Effects of tamoxifen or letrozole on lipid profile, vitamin $\mathrm{D}$ and estradiol serum levels in an obese postmenopausal woman with breast cancer. Int J Pharm Pharm Sci 2017;9(2):142-144. 